\title{
La Maison impériale japonaise et le principe de séparation de l'État et de la religion
}

\section{Éric Seizelet}

\section{(2) OpenEdition}

Édition électronique

URL : http://journals.openedition.org/rdr/1151

DOI : $10.4000 /$ rdr.1151

ISSN : 2534-7462

Éditeur

Presses universitaires de Strasbourg

\section{Édition imprimée}

Date de publication : 9 juillet 2020

Pagination : 159-171

ISBN : 979-10-344-0065-2

ISSN : 2493-8637

\section{Référence électronique}

Éric Seizelet, «La Maison impériale japonaise et le principe de séparation de l'État et de la religion », Revue du droit des religions [En ligne], 9 | 2020, mis en ligne le 18 mai 2020, consulté le 19 novembre 2020. URL : http://journals.openedition.org/rdr/1151 ; DOI : https://doi.org/10.4000/rdr.1151

\section{(c) (†) (8)}

La revue du droit des religions est mise à disposition selon les termes de la Creative Commons Attribution - Pas d'Utilisation Commerciale 4.0 International - CC BY-NC 4.0. 


\section{LAMAISON IMPÉRIALEJAPONAISE ETLEPRINCIPEDESÉPARATION DEL'ÉTATET DELARELIGION}

\section{ÉricSEIZELET}

Université Paris-Diderot

\section{RÉSUMÉ}

Après la suppression du shintô d'État dans la foulée de la défaite de 1945, la Constitution du Japon (1946) fournit le nouveau cadre juridique garantissant la liberté de religion en proclamant la séparation de l'État et de la religion. Cette évolution soulève d'importantes interrogations concernant la Maison impériale. Tout d'abord, du fait des liens étroits entre le shintô et la monarchie japonaise, la question se pose de savoir dans quelle mesure la liberté de croyance doit être reconnue à l'empereur et aux autres membres de la famille impériale en tant qu'individus. La seconde concerne l'interprétation par les gouvernements conservateurs du principe de neutralité religieuse de l'État, dans le contexte des cérémonies d'accession au trône suivant l'«abdication » de l'empereur Akihito.

\section{Abstract}

After the suppression of state Shinto in the wake of the 1945 defeat, the Constitution of Japan (1946) provided a new legal framework for religious freedom by separating the state from religion. This shift raised two important issues concerning the imperial House. Firstly, given the traditional links between the monarchy and Shinto as the national religion of Japan, to what extent freedom of religion has to be recognized to the emperor and the other members of the imperial family as individuals? The second interrogation is related to the interpretation by the conservative governments of the religious neutrality of the State in the context of the various events and rituals marking the enthronement ceremonies following the "abdication" of former emperor Akihito. 


\section{INTRODUCTION}

La fin du règne de Akihito, le $125^{\mathrm{e}}$ empereur à siéger sur le trône du Chrysanthème, le $1^{\text {er }}$ mai 2019 , et sa succession par son fils aîné, le prince impérial Naruhito, inaugurent pour la monarchie et le peuple japonais une nouvelle ère. Au sens propre comme au sens figuré. Cette transition monarchique - la seconde de cet «après-guerre » interminable - s'effectue cependant dans un contexte politique à bien des égards exceptionnel: le Japon, frappé par la triple catastrophe de Fukushima de mars 2011, est contraint de revoir un modèle de croissance qui s'appuyait, partiellement du moins, sur le développement de son parc nucléaire; les sept années de pouvoir continu du Cabinet Abe depuis 2012 - un record de longévité - ont été marquées par une offensive en règle contre l'actuelle Constitution de 1946, une réorientation de la politique de défense de l'archipel soulignant les capacités combattantes des forces d'autodéfense et une remise en scène du nationalisme à travers toute une série de crises touchant tant les questions mémorielles ( femmes de réconfort», manuels scolaires d'histoire, sanctuaire Yasukuni) que les contentieux territoriaux, anciens (Kouriles) comme nouveaux (Takeshima, Senkaku). Contexte exceptionnel aussi pour la monarchie: la succession au trône n'intervient pas à la suite des mécanismes légaux de dévolution prévus par le code de la Maison impériale, Kôshitsu tempan, de 1947, selon lesquels le décès de l'empereur est la seule et unique cause de succession, mais d'une «abdication» autorisée intuitu personae par le Parlement japonais à la suite de l'allocution télévisée du 8 août 2016 par laquelle Akihito - le futur empereur Heisei à son décès - annonçait à la nation, interloquée mais non surprise, son souhait de se retirer ${ }^{1}$. Une décision motivée par le grand âge et l'état de santé précaire du souverain, mais qui a donné lieu à de nombreuses spéculations sur ses implications - voire ses motivations - politiques. Et qui va donner le coup d'envoi à une longue période de transition - plus de trois ans - qui ne s'achèvera qu'à l'automne 2019, avec la Gustation solennelle des prémices, Daijôsai. Or, depuis l'automne 2018, la polémique enfle autour du respect par le gouvernement Abe du principe de séparation de l'État et de la religion dans l'organisation de la transition. D'où l'idée d'en interroger le fonctionnement et la portée à la lueur d'un double questionnement: la liberté de religion de la famille impériale d'une part et le régime juridique des cérémonies de succession d'autre part.

1. Loi spéciale $\mathrm{n}^{\circ} 63$ du 16 juin 2017 relative au code de la Maison impériale portant organisation de l'abdication de l'empereur. 


\section{LA LIBERTÉ DE RELIGION DES MEMBRES DE LA FAMILLE IMPÉRIALE APRÈS 1945}

\subsection{DE LA RECONNAISSANCE DE PRINCIPE À LA LIMITATION DE FAIT}

La défaite consommée et le shintô d'État ${ }^{2}$ éradiqué, les autorités d'Occupation ont reconnu à l'empereur, et donc à la famille impériale, la liberté de religion: dans cette perspective, il leur était possible d'adhérer au shintô et d'entretenir des relations spéciales avec les sanctuaires traditionnellement liés à la Maison impériale, tels que le Grand sanctuaire d'Ise, mais à condition qu'il s'agisse d'initiatives privées, purement individuelles, et non accomplies en tant que représentants de l'empereur ou de la famille impériale, et que ces activités religieuses ne soient pas liées à la position du monarque en tant que symbole étatique, tout en reconnaissant par ailleurs que la distinction entre la personne publique de l'empereur et sa personne privée était délicate à tracer. Dans cette optique, les autorités d'Occupation ont laissé intacts les rituels du Palais qu'elles distinguaient d'ailleurs du shint $\hat{o}^{3}$.

Si les nouvelles institutions démocratiques après 1945 fournissent un cadre propice à la liberté de religion, shinkyô no jiyû, la question demeure de savoir dans quelle mesure la famille impériale, compte tenu de son histoire et de sa position, bénéficie de la même protection constitutionnelle que le commun des citoyens énoncée par l'article 20 de la nouvelle loi fondamentale de $1946^{4}$. Pour le juriste catholique Tanaka Kôtarô (1890-1974), avec la disparition du shintô d'État, le peuple japonais «jouit désormais d'une liberté de religion pleine et entière». En conséquence, «l'empereur lui-même, est libéré des contraintes qui pèsent par exemple sur le roi d'Angleterre $»^{5}$. De ce fait,

2. On entendait par là la récupération des croyances nationales au profit du culte de l'empereur et des ancêtres impériaux afin de cimenter la cohésion psychologique et morale de la nation. H. Hardacre, Shintô and the State, 1868-1988, Princeton, Princeton University Press, 1989.

3. General Headquarters, Supreme Commander for the Allied Power, Summation of NonMilitary Activities in Japan, $\mathrm{n}^{\circ}$ 6, 1946, Boonjudo/8th U.S. Army Printing Plant, p. 238; W. K. Bunce, Religions in Japan: Buddhism, Shinto, Christianity from the Report Prepared by the Religions and Cultural Resources Division, Civil Information and Education Section, General Headquarters of the Supreme Commander for the Allied Powers, Vermont, Charles E. Tuttle, 1955, p. 170 ; S. SHimazono, Kokka shintô to nihonjin [Le shintô d'État et les Japonais], Tôkyô, Iwanami shoten, 2010, p. 185-186.

4. L'article 20 pose le principe de la liberté de croyance, de non-ingérence de l'État dans les affaires religieuses, et de neutralité politique des organisations religieuses. Il est doublé par l'article 89 qui interdit à l'État de financer des organisations et des activités à caractère religieux.

5. Le monarque britannique est en effet, depuis 1534, le chef de l'Église anglicane. 
«sur le plan institutionnel, il n'existe plus d'obstacle à son adhésion à la croyance qu'il estime la plus appropriée, suivant en cela ce que lui dicte sa conscience, en s'affranchissant de toute considération politique. Les libertés fondamentales concédées à l'empereur en tant qu'être humain ne sont en aucun point inférieures à celles qui sont reconnues à l'ensemble du peuple. L'empereur ne doit pas être sacrifié sur l'autel de la politique: la question de son bonheur et de son salut spirituel relève de la seule personne de l'empereur en tant qu'individu».

Néanmoins, sur la question de l'étendue de cette liberté, et notamment la possibilité de changer de religion, Tanaka est plus réservé: après avoir relevé que les conférences faites à l'empereur par des spécialistes des religions alimentaient, notamment dans la presse étrangère, les rumeurs de conversion «qui ne pouvaient qu'indisposer grandement Sa Majesté », Tanaka poursuivait:

«à supposer même que Sa Majesté soit parvenue, à titre personnel, à un certain degré de conviction, sa conversion soulève néanmoins des problèmes particulièrement délicats... Contrairement à l'époque de l'introduction du bouddhisme, en tant que symbole de la nation tout entière, il est désormais beaucoup plus difficile à l'empereur d'adhérer à une foi religieuse particulière. Il est impossible en effet de situer l'empereur sur le même plan que le Premier ministre qui, dans le cadre de la nouvelle Constitution, peut se maintenir en place quelle que soit son appartenance religieuse, car c'est un fait que la Maison impériale entretient des liens historiques profonds avec le shintô des sanctuaires, jinja shintô ${ }^{6} »$.

L'historien des religions Kishimoto Hideo (1903-1964), qui avait travaillé dans le cadre de la Civil Information and Education Section (CIE) sous l'Occupation américaine, synthétise la problématique à laquelle les membres de la famille impériale sont désormais confrontés. Après avoir souligné les symboles d'ouverture et d'humanité manifestés selon lui par l'éducation reçue par le prince héritier Akihito et la personnalité du prince impérial Mikasa (1915-2016) - le frère cadet de Hirohito (1901-1989), spécialiste de l'Ancien Testament - il poursuivait ainsi: «tout en reconnaissant les contraintes issues de la tradition et de la spécificité de sa condition, fondamentalement, la Maison impériale doit être libre en matière religieuse ${ }^{7} »$.

6. K. TANAKA, «Ai wa subete ni katsu» [L'amour l'emporte sur tout], cité par H. OnizukA in Tennô no rosario [Le chapelet de l'empereur], Tôkyô, Seikô shobô, 2015, vol. 1, p .112 et 114 [toutes les traductions sont de l'auteur].

7. Asahi shinbun, 21 nov. 1954. 
Ces prises de position ne sont cependant pas sans difficulté: la pétition de principe - la liberté de religion - y est aussitôt contextualisée par la référence aux contraintes de statut et de tradition qui pèsent sur la Maison impériale. Faut-il dès lors comprendre que la reconnaissance de ces contraintes, qui transcendent clairement la Constitution, imposent de facto aux individus membres de la famille impériale des restrictions quant à leurs croyances et à l'expression de leurs convictions religieuses?

\subsection{LA FAMILLE IMPÉRIALE ET LES DROITS ATTACHÉS À LA CITOYENNETÉ}

À première vue, les membres de la famille impériale jouissent de la même liberté de religion que n'importe quel autre citoyen, puisque celle-ci est garantie, selon la Constitution, à « tous». Pour la plupart des constitutionnalistes cependant, cette question fait partie d'une interrogation plus large, celle de l'applicabilité à la famille impériale du chapitre III de la loi fondamentale sur les droits des citoyens. La position officielle des pouvoirs publics consiste à soutenir que la famille impériale faisant partie du "peuple», kokumin, elle jouit par principe des mêmes droits, mais que du fait de la position de l'empereur en tant que "symbole de l'État et de l'unité du peuple ${ }^{8}$ » et des liens de parenté qui unissent les membres de la famille impériale à l'empereur - tennô en japonais -, ces droits subissent des restrictions, tant en ce qui concerne les libertés d'expression, d'opinion que les droits politiques accordés normalement aux citoyens ${ }^{9}$. En d'autres termes, le champ d'application ratione personae du chapitre 3 de la loi fondamentale dépendrait à la fois du degré de proximité à l'égard du trône - les contraintes pesant sur les ascendants et descendants en ligne directe étant plus importantes que pour les collatéraux - et de la nature des droits dont il s'agit. Ainsi, en matière de mariage, le principe d'égalité et de non-discrimination inscrit à l'article 14 ferait obstacle à la seule invocation d'un motif religieux pour entraver le mariage d'un membre de la famille impériale. L'exemple de l'ex-impératrice Michiko est à cet égard édifiant: originaire d'un milieu catholique, les Shôda, et ayant fait ses études à l'Université du Sacré-Cœur pour jeunes filles, ces antécédents n'ont pas été jugés suffisamment probants pour dissuader le gouvernement d'autoriser le prince héritier Akihito à l'épouser. Il est vrai que le catholicisme de la future impératrice n'était pas des plus ostentatoires même si ce choix, qui rompait avec la pratique des mariages endogamiques

8. Art. 1 de la Constitution de 1946.

9. Yoshikuni Ichirô, directeur du Bureau législatif du Cabinet, chambre des Conseillers, commission du Cabinet, 20 nov. 1975. 
dans la Maison impériale, faisait difficulté au sein même de la famille impériale $^{10}$. D'un autre côté, selon la «rumeur», ce serait l'Église catholique qui, dans une stratégie de pénétration de la Maison impériale, aurait fait pression pour que la future impératrice ne soit pas baptisée... Une thèse bien évidemment non étayée, mais doublement absurde au regard du droit canon et du simple bon sens.

En matière religieuse, la même ambiguïté existe: certes, les membres de la famille impériale jouissent des libertés de conscience et de pensée qui constituent en quelque sorte les prémisses de la liberté de religion. Comme pour l'ensemble des citoyens, du fait du principe de séparation de l'État et de la religion, seikyô bunri, les croyances religieuses de la famille impériale - la liberté de croire, de ne pas croire, ou de changer de religion - relèvent de l'intimité de la vie privée. Mais dès lors que cette liberté s'extériorise en actes et pratiques, elle subirait les restrictions précédemment évoquées en matière d'expression et d'opinion. En outre, il serait impossible, du fait du principe précité de séparation, à la Maison impériale d'exciper de ses propres croyances pour imposer ou introduire une connotation religieuse à des cérémonies nationales organisées par les pouvoirs publics ${ }^{11}$.

Pour l'empereur, la situation est même encore plus compliquée: si l'on considère que le monarque est bien un membre constitutif de la nation japonaise au même titre que la collectivité des citoyens japonais, les droits et libertés garantis par la Constitution lui sont appliqués de plein droit et les exceptions qui dérivent de son statut de symbole doivent être strictement interprétées. En revanche, si l'on considère que la loi fondamentale distingue clairement l'empereur, en tant que symbole, du peuple-citoyen souverain,

10. On mentionnera qu'au cours du Conseil de la Maison impériale réuni en novembre 1958, la question de la religion de la candidate fut néanmoins posée par le Premier ministre Kishi Nobusuke (1896-1987). Le directeur de l'agence de la Maison impériale indiqua qu'à partir du moment où $\mathrm{M}^{\text {lle }}$ Shôda n'avait pas été formellement baptisée, le mariage ne posait pas de problème. Constitutionnellement cependant, cette réponse fait difficulté: elle sous-entend que si $\mathrm{M}^{\text {lle }}$ Shôda avait été baptisée, le Conseil de la Maison impériale aurait pu statuer différemment.

11. H. NAGAO, «Kôkenryoku to shûkyô. Ôsaka-shi jizôzô soshô wo keiki to shite» [Puissance publique et religion. À l'occasion du procès concernant les statues de Jizo de la ville d'Ôsaka], Chûkyô hôgagu, vol. 28, n 3/4, 1994, p. 80. Contra A. MoмоchI, «Seikyô bunri to shinkyô no jiyû» [Le principe de séparation de l'État et de la religion et la liberté de religion], Kôhô kenkyû, vol. 32, n 321, 1990, p. 90. Pour une présentation plus générale des rapports entre l'État et la religion au Japon, M. Gotô, Seikyô bunri no kiso riron [Les principes fondamentaux de la séparation de l'État et de la religion], Tôkyô, Seibundô, 2018; D. O'Brien with Y. Ohkoshi, To Dream of Dreams: Religious Freedom and Constitutional Politics in Postwar Japan, Honolulu, University of Hawaii Press, 1996. 
alors le chapitre III de la loi fondamentale, qui ne vise que le peuple-citoyen, ne peut lui être appliqué; l'exception devient ici la règle, car la loi fondamentale entérinerait un statut dérogatoire, pour ne pas dire discriminatoire, dont l'empereur serait à la fois le bénéficiaire... et la victime. Quoi qu'il en soit, l'empereur ne pourrait opposer sa conscience ou ses croyances à l'accomplissement des «actes en matière de représentation de l'État»- les pouvoirs constitutionnels de l'empereur: il s'agirait là d'une position politique qui lui est proscrite par la Constitution ${ }^{12}$. Mais s'il le faisait, on se trouverait en face d'une impasse constitutionnelle, sauf à détourner les dispositions relatives à un empêchement provisoire du monarque...

Pourrait-il se convertir à une autre religion? Des rumeurs ont effectivement beaucoup circulé, sous l'Occupation, à propos d'une conversion possible de l'empereur Hirohito (Shôwa) au christianisme et, de façon plus générale, sur les liens entre la Maison impériale et cette religion. Cependant, souligne-t-on au Japon, à la différence de l'islam qui serait une religion extravertie - le croyant étant tenu d'attester jusque dans sa vie sociale les signes d'appartenance à la communauté musulmane - le christianisme serait, d'abord, une religion du for intérieur. Et dans ces conditions, nul besoin de conversion officielle. Nul besoin non plus de se rattacher à une quelconque Église ou obédience, afin de désamorcer les stratégies d'accaparement et de récupération. Rien n'empêcherait l'empereur de nourrir des convictions chrétiennes, à la façon d'un «chrétien caché», de recevoir en privé des pasteurs ou des prêtres - les précédents existent - voire de se faire subrepticement baptiser. Une information qui aurait tout de même du mal à rester confidentielle... Certes l'appartenance éventuelle de membres de l'entourage de la famille impériale au quakerisme bien implanté dans l'archipel, notamment sous l'Occupation, et qui pratique un christianisme sans dogme, hiérarchie et lieu de culte attitré, aurait pu faciliter des conversions privées, mais cette thèse n'est nullement corroborée. Il faut croire que même si la «conversion » de l'empereur au christianisme n'est plus d'actualité, la question a tout de même été évoquée, de façon officielle, en mars 1960, lors des travaux de la Commission d'investigation constitutionnelle du Cabinet. Son président,

12. Sur le plan international, le seul précédent récent dans une monarchie constitutionnelle se rapporte à l'attitude du roi des Belges Baudouin (1930-1993) qui avait refusé de signer, en mars 1990, par objection de conscience, la loi autorisant l'interruption volontaire de grossesse: le Parlement belge, à la suite d'une pirouette constitutionnelle, avait dû constater l'empêchement temporaire du roi pour promulguer la loi. Ce cas de figure est juridiquement impossible au Japon car d'une part l'empereur n'a pas de «pouvoirs de gouvernement», et d'autre part il n'a pas, en raison de ce fait même, de pouvoir de sanction législative. 
Takayanagi Kenzô (1887-1967) s'était interrogé sur la nature religieuse ou non du shintô et sur les répercussions d'une conversion possible de l'empereur. Itanuma Kazumi (1892-1982), ancien haut fonctionnaire du ministère de l'Intérieur, surtout ancien vice-président de la chambre des Affaires des dieux, jingi-in fukusôsai ${ }^{13}$, avait indiqué qu'une telle conversion serait un choc pour les milieux shintô, qu'elle attiserait la colère de l'extrême droite et créerait un climat de tension dans l'archipel comme celui qui avait opposé le clan des Mononobe à celui des Soga sur la question de l'introduction du bouddhisme ${ }^{14}$. Quelques années plus tard, en avril 1990, à la Diète, en réponse à une interpellation parlementaire sur la liberté de croyance de l'empereur, le vice-directeur de l'agence de la Maison impériale répondit par l'affirmative. L'échange qui s'ensuivit mérite d'être rapporté ${ }^{15}$ :

— «C'est grave! Est-ce à dire que si Sa Majesté dispose de la liberté de croyance. Elle pourrait librement adopter l'islam ou le christianisme comme religion?

— «Ma réponse n'était peut-être pas suffisante. Du fait que l'empereur est le symbole de l'État et de l'unité du peuple, Sa Majesté a toujours été attentive à accomplir les rites de la Maison impériale, kôshitsu no saishi, qui sont issus de la longue tradition de la Maison. La réponse que j'ai donnée tout à l'heure se situait à un niveau plus général. Et de ce point de vue, la liberté de religion, la liberté de croyance sont, juridiquement, des droits garantis au peuple [...] Sa Majesté fait preuve de la plus grande considération et attention pour les rites de la Maison Impériale accomplis conformément à cette longue tradition. »

Cette réponse reste embarrassante et non dénuée d'ambiguïté. D’abord elle interpelle la notion de symbole. Doit-on considérer que la position

13. Une structure de propagande et d'édification des masses créée au sein du ministère de l'Intérieur en novembre 1940, et supprimée en février 1946 dans la foulée de l'abolition du shintô d'État.

14. S. Mitsuchi, Yasukuni mondai no genten [À l'origine du problème du Yasukuni], Tôkyô, Nippon Hyôronsha, 2005, p. 153-154. Ce sont donc des raisons principalement politiques qui militeraient en faveur de la non-conversion. D'autres historiens japonais soulignent que la référence canonique à l'«essence nationale», kokutai est principalement liée à un principe de souveraineté tennocentré, mais dénué de doctrine et de vision du monde, que le shintô d'État n'a nullement entamé les convictions religieuses intimes de la famille impériale, et par conséquent qu'une éventuelle conversion impériale n'était pas suffisante, à elle seule, à altérer le kokutai du pays. Une position qui rejoint à la fois la position des Chrétiens sans Église sur la compatibilité des rites du palais avec le christianisme, mais aussi les positions «légalistes» d'un catholicisme respectueux des institutions existantes. T. HARA, Shôwa tennô jitsuroku wo yomu [Lire les Annales de l'empereur Shôwa], Tôkyô, Iwanami shoten, 2015, p. 195-196.

15. Miyao Iwao, Chambre des représentants, commission du Cabinet, 17 avr. 1990. 
symbolique de l'empereur en tant qu'institution est liée, de façon objective, à l'accomplissement des rites de la Maison impériale? Ou bien, de façon plus subjective, est-ce que ce sont les convictions religieuses propres du tennô qui l'incitent à accomplir ces rites en tant que symbole? Dans le premier cas, ces rites constitueraient un devoir lié à la position de symbole, indépendamment des convictions religieuses. Dans le second cas, ce sont ces mêmes convictions qui gouverneraient l'attitude de l'empereur. Ensuite, cette réponse interpelle la mise en abyme entre la personne publique kôjin de l'empereur et sa personne privée shijin, alors que juridiquement ces rites, du fait de la séparation de l'État et de la religion, quoique liés effectivement à la Maison impériale, sont désormais de nature privée. C'est là la thèse défendue par la doctrine dominante de droit public. Mais elle n'est pas partagée par l'agence de la Maison impériale pour laquelle les rites de la Maison impériale, ceux qui ont pour cadre les trois sanctuaires du Palais, figurent au nombre des «obligations publiques», Kômu, de l'empereur, au nom de la position constitutionnelle du tennô comme «symbole de l'État et de l'unité du peuple».

\section{LE STATUT DES CÉRÉMONIES DE SUCCESSION ET LE RÔLE DU DAIJÔSAI}

\subsection{LA SIGNIFICATION DE LA CÉRÉMONIE DE LA GUSTATION SOLENNELLE DES PRÉMICES}

La cérémonie dite de la «Gustation solennelle des prémices» clôture l'ensemble des rites et cérémonies qui scandent la transition monarchique au Japon et qui débutent par les rites de l'accession au cours duquel les trois trésors sacrés, sanshu no jingi - les regalia - sont remis au nouveau monarque. Elle se poursuit par les rites d'intronisation, Sokui no rei, par lesquels est officiellement proclamée la montée sur le trône du nouvel empereur en présence des «corps constitués » et des représentants étrangers. De l'ensemble de ce cycle de cérémonies, c'est celle du Daijôsai, célébrée dans la nuit du 14 au 15 novembre 2019, qui soulève le plus de difficultés au regard du principe constitutionnel de séparation de l'État et de la religion. En effet, s'il s'agit d'une cérémonie «religieuse», le principe de neutralité religieuse de l'État s'oppose, en vertu de l'article 89 de la loi fondamentale, à tout financement officiel. La caractérisation du Daijôsai en tant que cérémonie "religieuse» soulève néanmoins de nombreuses difficultés en raison du voile de mystère qui entoure tant la nature que le déroulement même de cette cérémonie.

Tout d'abord la «Gustation solennelle des prémices», à la différence des rites d'intronisation, n'est pas publique, même si elle fait l'objet d'invitations officielles. C'est une cérémonie nocturne qui se déroule dans un espace sacré, 
à l'ouest et à l'est duquel sont érigés, entre autres, deux pavillons en bois brut, le Yuki-den et le Suki-den. Le futur empereur qui, les jours précédents, a procédé à des rites de purification, revêtu de ses habits sacerdotaux immaculés, accède à cet espace sacré par une procession au cours de laquelle les prêtres shintô déroulent une sorte de natte, afin que ses pieds ne foulent pas directement le sol. C'est la seule partie de la cérémonie sur laquelle il existe des photographies. Le déroulement de la cérémonie elle-même est totalement secret. L'on sait seulement que dans ces deux pavillons, le nouvel empereur présente solennellement à la déesse tutélaire du clan impérial, AmaterasuÔmi-Kami et aux dieux, les prémices du Nouveau riz ainsi que les deux sake, noir et blanc, spécialement confectionnés à cet effet, en provenance de deux régions de l'ouest et l'est de l'archipel sélectionnées par divination. Cette offrande est suivie d'une consommation rituelle au cours de laquelle l'empereur partage ces mets avec les dieux. Anthropologues et spécialistes de l'histoire des religions se sont perdus en conjectures sur la signification à donner à ce rituel. D'où il se dégage malgré tout quelques constantes. En premier lieu, le Daijôsai rappelle la permanence des prérogatives sacerdotales de l'empereur. L'accomplissement des rites fait partie intégrante des attributions impériales alors même que les prérogatives politiques du tennô ont été souvent éclipsées. En second lieu, elles rappellent que dans une société agraire soumise aux rythmes, mais aussi aux caprices de la nature, les offrandes aux dieux sont à la fois une manifestation de reconnaissance et de prière pour les nouvelles récoltes et, qu'à travers cette fonction d'intercession, l'empereur est le garant suprême de la prospérité du pays. Le fait que le Daijôsai n'ait lieu qu'une fois par règne ne signifie pas que cette fonction est accomplie une fois pour toutes, mais qu'il renouvelle, à l'aube d'un nouveau règne, le lien consubstantiel entre la monarchie, la terre et les dieux. En troisième lieu, la Gustation solennelle des prémices est à la fois un rite de "communion » et de transmutation. En partageant le riz et le sake avec ses ancêtres divins, l'empereur est habité par l'esprit de ses prédécesseurs; il participe de leur nature et prend définitivement place dans la lignée impériale. En quatrième lieu, le Daijôsai est un rite de renaissance: héritier du trône par le sang, l'empereur émerge de la cérémonie en tant que tennô dûment investi. Certains anthropologues ont également souligné le caractère sexuel du Daijôsai, car les deux pavillons comportent une couche censée être le siège de la déesse solaire, mais ce point demeure encore controversé. Enfin, le Daijôsai instaure un rapport particulier au temps: non seulement par sa durée, mais aussi parce qu'il s'inscrit dans un espace éphémère et volontairement primitif. Éphémère, car les pavillons construits sont appelés à disparaître à l'issue de la cérémonie: l'instant de cette rencontre intime avec les divinités est 
exceptionnel donc fugace. Primitif, car les matériaux, procédés de construction et ustensiles utilisés reproduisent des techniques immémoriales: leur rusticité est à l'image d'une monarchie issue du fond des âges ${ }^{16}$.

\subsection{LE RÉGIME JURIDIQUE DES CÉRÉMONIES DE DÉVOLUTION}

Dès lors quel statut accorder juridiquement à ces rites? Si la Constitution japonaise, dans son article 7 alinéa 10 énonce au nombre des «actes en matière de représentation de l'État», soumis à l'avis et à l'approbation du Cabinet, l'accomplissement des "cérémonies», Gishiki, la pratique institutionnelle et la doctrine dominante s'accordent à dire que les «cérémonies» en question sont purement protocolaires: remise de décorations ou nominations par exemple. Le code de la Maison impériale de 1947 n'évoque que très sommairement, dans ses articles 24 et 25, l'existence de deux cérémonies: celles de l'intronisation précitée et des funérailles impériales. Toutes liées à la dévolution. De ce fait, il est concevable de prévoir pour ces cérémonies une prise en charge par l'État, mais dans les limites du principe précité de séparation. Toutefois, le laconisme du législateur pose deux types de problèmes car l'organisation et la pratique de ces cérémonies s'inspirent dans les faits des précédents et des réglementations du Japon impérial ${ }^{17}$. De plus, se pose la question de savoir si le législateur, en mentionnant les deux cérémonies précitées, a entendu exclure toutes les autres cérémonies et rituels du champ d'une prise en charge officielle, ou distinguer seulement ceux pour lesquels cette dernière était obligatoire. En d'autres termes, le gouvernement peut-il classer en tant qu'actes en matière de représentation de l'État tout ou partie des cérémonies non explicitement mentionnées par la loi? La cérémonie de la Gustation solennelle des prémices, qui n'est citée ni dans la Constitution ni dans le code précité, doit faire face à cette double interrogation. Certains constitutionnalistes, tels que Kobayashi Setsu, professeur honoraire à l'Université Keiô, soutiennent qu'à partir du moment où la loi fondamentale admet l'existence d'une dérogation majeure au principe d'égalité avec le statut particulier fait à la Maison impériale et à ses membres, rien n'empêche à ce qu'elle tolère une semblable exception pour le Daijôsai, dans la mesure où

16. Sur l'historique de ces cérémonies, les travaux de François MAcÉ font autorité: «Les rites d'avènement au Japon. L'invention d'une tradition $»:$ http://inalcocej.free.fr/travaux/ documents/ciplpdf/Cip.1.3.Mace.pdf [consulté le 27 janv. 2020].

17. Et notamment de l'ordonnance de la Maison impériale $\mathrm{n}^{\circ} 1$ du 11 février 1909 portant organisation des cérémonies de dévolution, formellement abrogée le $1^{\text {er }}$ mai 1947 avec l'entrée en vigueur de la Constitution. 
ce rite, dont la nature religieuse n'est pas contestée, fait partie intégrante des rites de succession au trône dont l'existence même est reconnue par la Constitution. Le gouvernement a fait savoir, de son côté, que conformément au précédent de 1989-1990, les rites de l'avènement seront considérés comme «actes en matière de représentation de l'État» et financés sur le budget de l'État. En revanche, certains rituels particuliers, comme le rapport aux dieux du changement de règne dans les trois sanctuaires du Palais n'auront pas de caractère public, en raison de leur connotation religieuse. C'est cette même raison qui justifie que la Gustation solennelle des prémices n'est pas classée au nombre des actes en matière de représentation de l'État. Mais elle bénéficiera néanmoins de fonds publics pris sur les « dépenses du palais », Kyûteihi, «en raison de son importance capitale dans les cérémonies traditionnelles de dévolution et de succession au trône ${ }^{18}{ }_{»}$. En conséquence, 2,7 milliards de yens ont été provisionnés pour cette cérémonie. Cette décision ne fait pas toutefois l'unanimité: des organisations religieuses, chrétiennes et bouddhistes craignent que ces cérémonies fournissent un prétexte au gouvernement pour réactiver le shintô d'État au nom du respect des traditions. Le prince impérial Fumihito, alors en seconde position dans l'ordre de succession a, en novembre 2018, émis des doutes sur la constitutionnalité de la position du gouvernement Abe, eu égard au caractère hautement religieux de l'événement, qu'il aurait préféré voir financé sur la liste civile et regretté que le directeur de l'agence de la Maison impériale n'ait pas retenu sa proposition d'utiliser l'un des trois sanctuaires du palais, le Shinka-den, qui aurait été tout à fait approprié à cet effet. Une déclaration, sans précédent, que même ceux qui partagent cette même ligne ont jugé problématique car sortant de la réserve qui s'impose à tout héritier présomptif, et qui va relancer, à coup sûr, la question des droits et libertés politiques des membres de la famille impériale ${ }^{19}$.

\section{CONCLUSION}

L'appartenance à la Maison impériale n'est pas incompatible avec des manifestations d'intérêt à l'égard du fait religieux, à la réception de dignitaires religieux, à la visite d'établissements de culte, ou au soutien à des activités caritatives organisées par des organisations religieuses. De nombreux

18. Yamamoto Shin'ichirô, directeur de l'agence de la Maison impériale: Mainichi shinbun, 30 mars 2018.

19. Asahi shinbun, Yomiuri shinbun, 30 nov. 2018; Mainichi shinbun, $1^{\text {er }}$ déc. 2018. 
précédents existent à cet égard depuis l'époque de Meiji. Mais étant donné la nature même de la monarchie japonaise et de ses liens avec le shintô, toute idée de «conversion» de la famille impériale à une autre croyance constituerait un séisme politique et culturel qui fragiliserait l'institution impériale elle-même. Comme après la disparition de Hirohito en janvier 1989, il est probable que la justice japonaise sera appelée à se prononcer sur la constitutionnalité des cérémonies de succession ${ }^{20}$. La Cour suprême a considéré dans le passé que l'assistance de gouverneurs ou de représentants de collectivités locales à ces cérémonies ne contrevenait pas au principe de neutralité religieuse au motif qu'il ne s'agissait que d'une «convenance sociale de caractère protocolaire », tenant compte de la position constitutionnelle du tennô, n'ayant «ni pour but, ni pour effet, de favoriser une religion particulière», selon une formule consacrée par la Haute juridiction dans son arrêt de juillet $1977^{21}$. Il est possible même que ces cérémonies soient contestées, non pas seulement sur la question du financement, mais sur le fond et leur signification: les trois insignes de la monarchie sont, selon la tradition, d'origine divine: le soutien gouvernemental aux cérémonies de transfert ne fournit-il pas une caution officielle aux récits mythologiques sur l'origine de la dynastie? Quelle que soit la réponse, il n'en demeure pas moins significatif de constater qu'ont survécu, après 1945, des manifestations et pratiques en matière de transition monarchique héritées de l'ancien régime, et plus particulièrement de l'époque de Meiji, où la plupart de celles-ci ont été «shintôisées» à des fins politiques. Et que le Japon démocratique s'est trouvé, et se trouve encore, dans l'incapacité de définir des protocoles de succession au trône adaptés à une monarchie constitutionnelle appuyée sur le principe de souveraineté populaire.

20. Sur les premières actions en justice devant le tribunal de district de Tôkyô : Asahi shinbun, 10 déc. 2018.

21. Cour suprême, ass. restreinte, 9 et 11 juill. 2002, 28 juin 2004: Minshû, vol. 56, no 6, p. 1204; Shûmin, no 253, p. 23; ass. plénière, 13 juill. 1977: Minshû, vol. 31, n 4, p. 533, à propos du financement public de la cérémonie de pose de la première pierre d'un gymnase municipal. Parmi les autres sujets de controverse concernant le principe de neutralité religieuse de l'État, on note les visites des membres du gouvernement au sanctuaire Yasukuni, un haut lieu du shintô d'État avant 1945, et l'entretien par l'État des tombes et mausolées impériaux. 